\title{
Neonatal and Two-Stage Olfactory Bulbectomy: Effects on Male Hamster Sexual Behavior
}

\author{
SARAH S. WINANS and J. BRADLEY POWERS ${ }^{1}$ \\ Department of Anatomy and Neuroscience Laboratory, \\ University of Michigan, \\ Ann Arbor, Michigan 48104
}

\begin{abstract}
The copulatory behavior of sexually mature male hamsters was investigated following removal of the olfactory bulbs in ways which might be expected to minimize postoperative behavioral effects. Neither two-stage bulbectomy in adults nor a one-stage operation in neonates prevented the total abolition of sexual behavior consequent to bilateral olfactory bulb removal in most of the animals. However, two bulbectomized hamsters (one, one-stage; one, two-stage) displayed an atypical persistence of mating postoperatively. Their lesions, which included total bilateral destruction of the main and accessory bulbs and damage to adjacent olfactory tissue, were histologically comparable to the lesions in two other bulbectomized hamsters (from different studies) which also copulated postoperatively. The behavior of these atypical animals supports the hypothesis that male hamsters can display normal sexual behavior in the absence of olfactory stimulation.
\end{abstract}

Male hamsters fail to copulate following olfactory bulbectomy (Murphy and Schneider, 1970). Whether this behavioral deficit is due to anosmia alone, to a neurological deficit not dependent on olfactory sensation, or to some combination of these factors, has not been resolved. We have demonstrated that hamsters continue to initiate sexual behavior after being made anosmic by intranasal perfusion of a zinc sulfate solution (Powers and Winans, 1973). This finding suggested to us that the olfactory bulbs contribute to the integrity of sexual behavior mechanisms in addition to, and independent of, their role in processing sensory information. However, other laboratories have obtained conflicting results and on the basis of their findings have asserted that the elimination of mating behavior after olfactory bulb removal reflects a sensory deficit only. These conclusions are based on the failure of male hamsters to mount estrous females after being made peripherally anosmic by

${ }^{1}$ We wish to thank Elliot Valenstein for helpful discussions throughout this investigation and Trish Britten and Denise Fleming for assisting in behavioral testing and histological preparation. Research supported by NIMH Grant No. MH20811 to E.S.V. 
exposing the olfactory epithelium to either zinc sulfate (Lisk, Zeiss, and Ciaccio, 1972) or procaine hydrochloride (Doty and Anisko, 1973), or by a technique involving unilateral olfactory bulb removal combined with clamping shut the external naris on the contralateral side (Devor and Murphy, 1973).

In all these studies, the treated animals' ability to smell is obviously a crucial variable. Only in our study was the anosmic condition of the hamsters tested by olfactory discrimination techniques rather than by odor preference methodology. Our zinc sulfate-treated animals totally lost the ability to identify the presence of either amyl acetate or female hamster estrous odor, yet at the same time they displayed normal sexual behavior in formal mating tests. These discrepancies in the literature prompted us to explore different approaches to this problem which might help resolve the controversy.

We decided to remove the olfactory bulbs in male hamsters with two techniques which might result in minimal behavioral deficits, although the animals would obviously be rendered completely anosmic. Evidence was available suggesting that in some cases the behavioral effects resulting from a single bilateral lesion performed in fully mature individuals could be remarkably attenuated if the lesion were made in two stages rather than one, or in one stage in the neonatal period (Greene, Stauff, and Walters, 1972; Nonneman and Isaacson, 1973; Schneider, 1970; Stein et al, 1969).

Prior to initiating our experiments, we learned that Fleming and Rosenblatt (1973) had produced a facilitation of maternal responsiveness in virgin female rats by making them peripherally anosmic with intranasal zinc sulfate treatment or by bulbectomizing them in two stages separated by $1 \mathrm{wk}$. However, when bilateral bulbectomy was performed in one stage, maternal aggressiveness and cannibalism ensued in most cases. These findings suggested that two-stage removal of the olfactory bulbs had altered the behavioral effects of the one-stage operation and had produced animals behaviorally comparable to the zinc sulfate-treated, peripherally anosmic females. This evidence encouraged our use of the two-stage operative procedure on the olfactory system of the male hamster. Recently, this technique has also been used in assessing olfactory system contributions to the control of sexual behavior in male mice (Rowe and Smith, 1973). The findings presented below indicate that neither two-stage bulbectomy in adulthood nor one-stage neonatal bulbectomy prevented the loss of copulatory behavior in male hamsters.

\section{EXPERIMENT 1}

\section{Methods}

Adult male hamsters obtained from Con Olson Co. (Madison, Wisc.) lived in individual cages in rooms with a reversed illumination schedule (lights off between 9 AM and 9 PM). Purina lab chow and water were available ad lib. 
Observations of mating behavior were conducted in clear Plexiglas arenas measuring $24 \mathrm{~cm}$ wide $\times 30 \mathrm{~cm}$ long $\times 21 \mathrm{~cm}$ high. Each male was adapted to the arena for $10 \mathrm{~min}$ prior to the introduction of an estrous female. The females had been ovariectomized and brought into behavioral estrus with subcutaneous injections of estradiol benzoate $(10 \mu \mathrm{g})$ and progesterone $(200 \mu \mathrm{g})$ administered 42 and $6 \mathrm{hr}$, respectively, prior to the test. Each female was checked for receptivity with a stud male before the testing session with the experimental male began.

Male hamster mounts, intromissions, and ejaculations during a 10-min test period were recorded on a Rustrak event recorder. The latency to first intromission, measured from introduction of the female (IL), number of intromissions preceding each ejaculation (I/E), and latency to ejaculation, also measured from introduction of the female (EL), were determined.

Surgery was performed on hamsters anesthetized with Nembutal $(75 \mathrm{mg} / \mathrm{kg}$ body weight). Animals were placed in a stereotaxic instrument and the frontal bone over the olfactory bulb(s) to be aspirated was removed by thinning with a dental bur. In sham operations the dura was not intentionally incised, although in several animals it was inadvertently cut without damage to the underlying olfactory bulb. Brain tissue was removed by suction with a pipette and the olfactory fossa was packed with Gelfoam before the incision was sutured.

The effects of two-stage bulbectomy were investigated in animals which were permitted minimal sexual experience between operations (Set I) as compared to animals which were allowed several opportunities for copulation in the interval between removal of the first and second olfactory bulb (Set II).

Set I was comprised of three groups which were equated for mean EL scores achieved on a preliminary mating test 2 days prior to the first operation. Animals which failed to achieve two ejaculations within $5 \mathrm{~min}$ on this pretest were eliminated from the experiment. The three groups of Set I were restricted to a single mating experience between operations and each received a different surgical treatment as depicted in Fig. 1. Following the second operation the mating behavior of the animals which had sustained two-stage lesions (Group A) was compared to the behavior of hamsters in

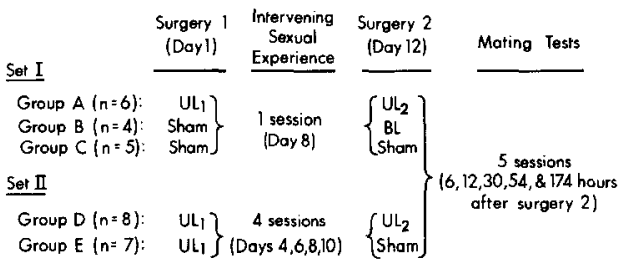

Fig. 1. Protocol for Expt. 1. $\mathrm{UL}=$ unilateral bulbectomy; $\mathrm{BL}=$ bilateral bulbectomy. 
which the bulbs had been removed in one stage (Group B) and to that of sham-operated controls (Group C).

The hamsters in Set II were given four separate mating opportunities in the 12-day interval between operations. This set was comprised of two surgical treatment groups (Groups D and E) which were also equated for mean EL on a preliminary mating test 2 days prior to the first operation. Bulbectomy was performed in two stages on the animals of Group D. Group E hamsters were unilaterally bulbectomized at the first operation, but sham operated at the time of the second surgery (Fig. 1).

The interoperative copulatory experience of animals in both Sets I and II was provided by giving mating tests according to the procedures described above. After the second operation mating behavior tests were conducted at 6 , $12,30,54$ ( 2 days), and $174 \mathrm{hr}$ ( 7 days). Observations were made frequently and at these early postoperative intervals because of evidence we had obtained from a pilot study in which several two-stage bulbectomized males had continued to mate for a brief period after the second operation, but had stopped mating after 2-3 days.

In order to determine whether an interoperative interval longer than 12 days and including more than four sexual experience sessions would promote mating after the second operation of a two-stage procedure, the animals of Group E were given additional treatments. These hamsters had had a unilateral bulbectomy, four interoperative tests, and the five regular behavior tests after the sham operation. They were then provided with four additional mating opportunities spaced over the next 28 days before the second olfactory bulb was removed. These males, therefore, had had a total of 13 mating sessions over a 45-day period which intervened between the two stages of the bulbectomy procedure. Following removal of the second olfactory bulb, their mating behavior was tested at $6,12,30$, and $54 \mathrm{hr}$.

After the final behavior test, all bulbectomized hamsters were anesthetized and perfused intracardially with $0.8 \% \mathrm{NaCl}$ followed by neutral formalin $(10 \%)$ in $0.8 \% \mathrm{NaCl}$. The brains were exposed by removal of all bone except the frontal and ethmoid surrounding the frontal poles and olfactory peduncles. After decalcification in 5.0\% formic acid the tissues were embedded in egg yolk and gelatin, frozen, sectioned at $40 \mu \mathrm{m}$, and stained with cresyl violet for analysis of the lesions.

\section{Results}

After unilateral bulbectomy ( $\mathrm{UL}_{1}$ ) all five males in Group A achieved two ejaculations in the intervening test session, but none of these hamsters showed mounts, intromissions, or ejaculations on any of the mating tests following the second bulbectomy $\left(\mathrm{UL}_{2}\right)$. Similarly, in Group $\mathrm{D}(n=8)$, all males mated normally during the mating experience sessions after $\mathrm{UL}_{1}$, but 
only one of the eight animals showed any mating behavior after $\mathrm{UL}_{2}$. With the exception of this one hamster, which mated on two of the five postoperative tests, removal of the olfactory bulbs in two stages abolished the sexual behavior of animals with either one (Group A) or four (Group D) copulatory experiences in the 12-day interval between operations. The behavior of all of these two-stage lesioned animals was essentially identical to that of hamsters in which the olfactory bulbs were removed simultaneously (Group B). In this group, one of the four males mated normally on four out of five postoperative mating tests, whereas the remaining three displayed no sexual behavior (see Figs. 2 and 3).

In contrast, all sham-operated controls (Groups $\mathrm{C}$ and $\mathrm{E}$ ) mated during three of the five behavior tests after the second operation. The decreased number of control animals ejaculating on the 54-hr test was probably similar to an exhaustion phenomenon. All of these males had mated normally on two (Group E) or three (Group C) separate mating tests within the previous 2 days and $100 \%$ of these animals ejaculated 5 days later on the 174-hr test. Hamsters of Group $E$ which were continued beyond the sham operation to a total of 13 mating sessions in the 45 days between $\mathrm{UL}_{1}$ and $\mathrm{UL}_{2}$ also failed to display copulatory behavior after the removal of the second bulb (Fig. 4).

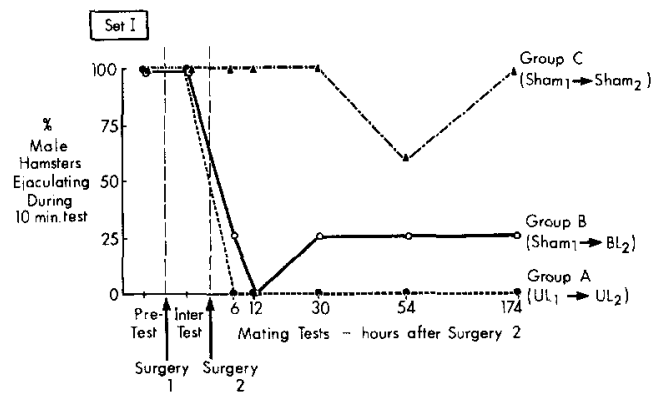

Fig. 2. Sexual performance of male hamsters bulbectomized in two stages (Group A: $n=6$ ) or in one stage (Group B: $n=4$ ) compared to sham operates (Group C: $n=5$ ).

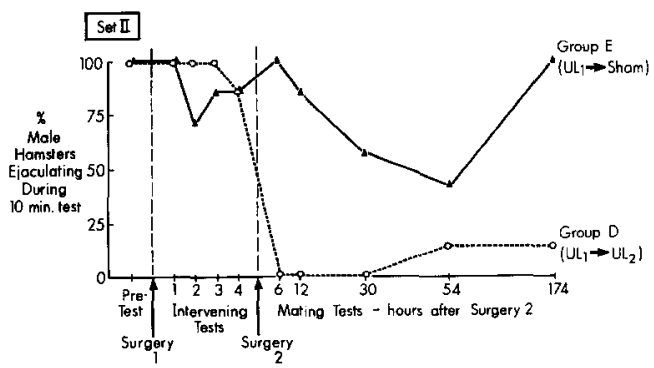

Fig. 3. Sexual performance of male hamsters bulbectomized in two stages (Group D: $n=8$ ) compared to unilaterally bulbectomized hamsters (Group E: $n=7$ ). 


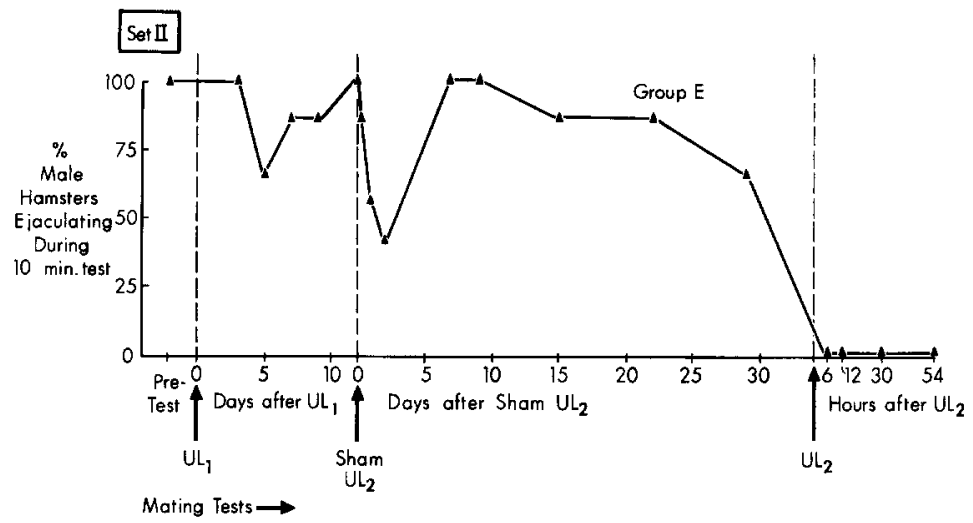

Fig. 4. Sexual performance of male hamsters given extended mating experience to a total of 13 sessions during the 45 days which intervened between $\mathrm{UL}_{1}$ and $\mathrm{UL}_{2}(n=7)$.

Histological analysis of the lesions revealed that the main olfactory bulbs (MOB) and accessory olfactory bulbs (AOB) had been completely removed in 18 of the 20 hamsters which received two-stage bulbectomies (Groups A, D, and $\mathrm{E}$ ). In the two animals with incomplete lesions only $5 \%$ of one MOB remained. (In one of these animals $\mathrm{AOB}$ was intact on the same side as the remaining MOB.) Neither of these hamsters with residual olfactory bulb tissue showed any mating behavior after the second operation. In contrast, the brain of the one two-stage hamster which mated after $\mathrm{UL}_{2}$ (see Group D in Fig. 3) showed $100 \%$ removal of $\mathrm{MOB}$ and $\mathrm{AOB}$ bilaterally.

Simultaneous bilateral bulbectomies performed on animals of Group B were complete (all of $\mathrm{MOB}$ and $\mathrm{AOB}$ destroyed) in two of the four animals, and $95 \%$ of both bulbs had been removed in the other two males. Again, neither of the animals with remaining olfactory bulb tissue mated during the postoperative testing sessions, but one of the hamsters with a complete bilateral lesion achieved ejaculations on four of the five tests (see Group B in Fig. 2). The sexual behavior observed in this animal from the one-stage group (B) and in one male from the two-stage group (D) appeared to be comparable to that of the sham-operated controls (Table 1).

The brains of all lesioned hamsters showed some damage to the anterior olfactory nucleus. In addition the majority of animals had sustained very slight damage to one or both frontal poles and in half of the animals the most anterior part of the olfactory tubercle showed some evidence of injury.

\section{EXPERIMENT 2}

As a second approach to minimizing the behavioral effects of olfactory bulb removal, we bulbectomized male hamsters neonatally. Although the 
TABLE 1

Postoperative Sexual Behavior of Two Atypical Bulbectomized Hamsters Compared to Control Animals

\begin{tabular}{llcc}
\hline & \multicolumn{3}{c}{ Mean performance scores $^{a}$} \\
\hline & IL & I/E & EL \\
Groups C \& E (Shams) & 76.2 & 9.5 & 166.0 \\
H-424 (two stage) & 97.5 & 9.5 & 181.0 \\
H-396 (one stage) & 69.0 & 9.5 & 161.3 \\
\hline
\end{tabular}

$a_{\mathrm{IL}}=$ intromission latency $(\mathrm{sec}) ; \mathrm{I} / \mathrm{E}=$ intromissions to ejaculation; $\mathrm{EL}=$ ejaculation latency $(\mathrm{sec})$. Mean values based only on tests positive for ejaculation: shams, $5 / 5$; $\mathrm{H}-424,2 / 5 ; \mathrm{H}-396,4 / 5$.

effect of this particular procedure on the social behavior of female hamsters has been reported (Leonard, 1972), a similar approach with males of this species has not yet been investigated.

\section{Methods}

Fourteen pregnant females from Con Olson Co. lived in breeding cages with Purina Lab chow and water available ad lib; occasional supplements of sunflower seeds and apples were provided. Following parturition, females and their litters were left undisturbed for 12 days. On Day 12 all males were given a bilateral bulbectomy. Surgery was performed under cryogenic anesthesia by immersion in ice; the frontal bone over the olfactory bulbs was gently removed and the olfactory tissue aspirated with 23-gauge hypodermic tubing. After packing the fossa with Gelfoam, the skin incision was sutured and the animals placed under a heat lamp to recover. When operated males were returned to their respective litters, sufficient females were sacrificed to ensure that there were no more than eight animals per litter. On Day 22 females were weaned, but the males remained with their mothers for 4 additional days. On Day 26 the bilaterally bulbectomized males were placed in social groups of two such males and two intact females, none of which was necessarily a littermate, although this arrangement was not purposely avoided. This social grouping was established to provide continual opportunities for social interaction between the operated males, and females which would be sexually receptive to these males every 4 days after puberty unless a pregnancy or pseudopregnancy ensued. These groups of four animals were housed in standard Wahmann cages $40.64 \mathrm{~cm}$ wide, $25.40 \mathrm{~cm}$ deep, and $17.78 \mathrm{~cm}$ high with food and water available ad lib. We determined independently that this cage size was sufficiently large to permit copulation. Beginning when the males were approximately 40 days old, all group cages were periodically examined specifically for the presence of hamster pups 
which would have provided unequivocal evidence that one or more of the neonatally bulbectomized males was capable of normal copulatory activity. Males and females remained together until the males had reached 105 days of age.

On Days 105 and 115 each male was given a $10-\mathrm{min}$ sex behavior test. During the 10-day interval between tests these males were removed from their group cages and housed individually. The procedures used for observing sexual behavior and for subsequently determining the completeness of olfactory bulb removal were identical to those described for Expt. 1.

After neonatal surgery and prior to weaning, 19 of 35 bilaterally bulbectomized males either died or were cannibalized. Two additional animals died after weaning, thus 14 hamsters were tested for adult sex behavior.

\section{Results}

None of the neonatally bulbectomized hamsters showed any evidence of copulatory behavior during the two standard mating tests given in adulthood. During the test periods these males either ignored the female or spent a significant portion of the test session in persistent biting and/or mouthing of the female's tail. This behavior is not unique to males bulbectomized neonatally; we have occasionally observed this pattern of responding in males which were bilaterally bulbectomized as adults.

At no time did the periodic inspection of living cages indicate that any hamster pups had been delivered. On some occasions females were observed in the lordosis posture although the males were not mounting. Presumably, this female sexual response was induced by the normal bodily contact provided in the social group.

Histological evaluation of the lesions showed that the main and accessory olfactory bulbs had been completely removed in 12 of the 14 hamsters. Most brains showed some damage to the anterior olfactory nucleus and slight encroachment of the lesion into the frontal poles, but no damage to the olfactory tubercle was present.

\section{Discussion}

Male hamsters failed to display normal patterns of sexual behavior following removal of the olfactory bulbs regardless of whether the operation was done in one or two stages (Expt. 1, Set I). Increasing the amount of sexual experience provided between the successive phases of the two-stage procedure in no way altered this result (Expt. 1, Set II). Additionally, male hamsters bulbectomized neonatally and provided extensive social experience during development also displayed mating behavior deficits when tested in adulthood (Expt. 2). 
Two hamsters in Expt. 1 continued to mate following bulbectomy, although in neither case did subsequent histological examination indicate that any main or accessory olfactory bulb tissue had been spared. Because one of the males was administered a one-stage procedure, and the second a two-stage procedure, it is unlikely that the technique of removing olfactory tissue in successive rather than simultaneous operations was relevant to this sparing of behavioral function.

Our findings that neither two-stage olfactory bulbectomy in adults nor one-stage bulbectomy in neonates attenuated the consequences of olfactory bulb removal is paralleled by other reports which demonstrate that these procedures do not always result in behavioral recovery. For example, the effects of dorsolateral prefrontal cortex lesions on various learning tasks in rhesus monkeys are minimized by a multiple-stage operative procedure (Rosen, Stein, and Butters, 1971), but this is not the case when orbital prefrontal cortex is similarly lesioned (Butters, et al. 1973). In addition, neonatal lesions of prefrontal cortex may or may not attenuate the behavioral consequences of the lesion depending on the cortical area involved (Goldman, 1971). In general, sparing or recovery of function following these procedures has been shown to be dependent upon the particular neural system damaged (Johnson, 1972). In this context, then, our results on the mating behavior of male hamsters may not be surprising.

In contrast to our findings with hamsters, the sexual behavior deficit in male mice resulting from olfactory bulb removal (Rowe and Edwards, 1972) can be significantly attenuated by a two-stage operative procedure similar to the one employed here (Rowe and Smith, 1973). But in accordance with our results (Powers and Winans, 1973), male mice made peripherally anosmic by zinc sulfate treatment copulate normally (Rowe and Smith, 1972). Such results suggest that fundamental differences may exist between mice and hamsters in the mechanisms by which the olfactory system contributes to the regulation of reproductive processes.

Investigations of the effects of olfactory ablation in females of these species support this suggestion. Female hamsters without olfactory bulbs show normal estrous cycles (Leonard, 1972) and lordosis behavior (Carter, 1973) and are not impaired in behavioral responsiveness to gonadal hormones following ovariectomy (Powers and Winans, unpublished). On the other hand, bulbectomized female mice have disrupted or irregular estrous cycles (Vandenbergh, 1973) and their behavioral receptivity mechanisms are refractory to exogenous estrogen and progesterone (Thompson and Edwards, 1972). This evidence is not conclusive, but it is consistent with the possibility that the olfactory system may interact with behavioral mechanisms differently in the two species.

We must consider the possibility that two-stage or neonatal bulbectomy in hamsters does minimize the nonsensory effect of olfactory bulb removal 
and that the absence of mating behavior which we observed was due to the animals' inability to smell. Although our original expectation that two-stage bulbectomized hamsters would continue mating was not confirmed, data from two animals reported here and two other hamsters from related studies in our laboratory support the hypothesis that the ability to smell is not a necessary condition for sexual behavior to occur in this species. In three of these four cases, no remnants of main or accessory bulb tissue were evident on histological examination; the fourth hamster had a small piece of main bulb remaining in the olfactory fossa, but this tissue was clearly detached from the peduncle posteriorly. In spite of this absence of olfactory bulb tissue, these males continued to display normal copulatory behavior following the operation for intervals of 2-14 days. We did not test the olfactory capabilities of these bulbectomized males, but there is no reason to believe that estrous odors could still be detected in the complete absence of olfactory bulb tissue. Because these animals continued to mate postoperatively, olfactory sensation cannot be essential for the initiation of normal copulatory behaviors. However, we in no way wish to deny that in the natural habitat of hamsters, olfactory stimulation may play an important role in controlling reproductive mechanisms.

\section{REFERENCES}

Butters, N., Butter, C., Rosen, J., and Stein, D. (1973). Behavioral effects of sequential and one-stage ablations of orbital prefrontal cortex in the monkey. Exp. Neurol. 39, 204-214.

Carter, C. S. (1973). Olfaction and sexual receptivity in the female golden hamster. Physiol. Behav. 10, 47-51.

Devor, M., and Murphy, M. R. (1973). The effect of peripheral olfactory blockade on the social behavior of the male golden hamster. Behav. Biol. 9, 31-42.

Doty, R. L., and Anisko, J. J. (1973). Procaine hydrochloride olfactory block eliminates mounting in the male golden hamster. Physiol. Behav. 10, 395-397.

Fleming, A., and Rosenblatt, J. S. (1974). Olfactory regulation of maternal behavior in rats. II. Effects of peripherally-induced anosmia and lesions of the lateral olfactory tract in pup-induced virgins. J. Comp. Physiol. Psychol. (in press).

Goldman, P. S. (1971). Functional development of the prefrontal cortex in early life and the problem of neuronal plasticity. Exp. Neurol. 32, 366-387.

Greene, E., Stauff, C., and Walters, J. (1972). Recovery of function with two-stage lesions of the fornix. Exp. Neurol. 37, 14-22.

Johnson, D. A. (1972). Developmental aspects of recovery of function following septal lesions in the infant rat. J. Comp. Physiol. Psychol. 78, 331-348.

Leonard, C. M. (1972). Effects of neonatal (day 10) olfactory bulb lesions on social behavior of female golden hamsters (Mesocricetus auratus). J. Comp. Physiol. Psychol. 80, 208-215.

Lisk, R. D., Zeiss, J., and Ciaccio, L. A. (1972). The influence of olfaction on sexual behavior in the male golden hamster (Mesocricetus auratus). J. Exp. Zool. 181, 69-78. 
Murphy, M. R., and Schneider, G. E. (1970). Olfactory bulb removal eliminates mating behavior in the male golden hamster. Science 167, 302-303.

Nonneman, A. J., and Isaacson, R. L. (1973). Task dependent recovery after early brain damage. Behav. Biöl. 8, 143-172.

Powers, J. B., and Winans, S. S. (1973). Sexual behavior in peripherally anosmic male hamsters. Physiol. Behav. 10, 361-368.

Rosen, J., Stein, D., and Butters, N. (1971). Recovery of function after serial ablation of prefrontal cortex in the rhesus monkey. Science 173, 353-356.

Rowe, F. A., and Edwards, D. A. (1972). Olfactory bulb removal: Influences on the mating behavior of male mice. Physiol. Behav. 8, 37-41.

Rowe, F. A., and Smith, W. E. (1972). Effects of peripherally induced anosmia on mating behavior of male mice. Psychonom. Sci. 27, 33-34.

Rowe, F. A., and Smith, W. E. (1973). Simultaneous and successive olfactory bulb removal: Influences on the mating behavior of male mice. Physiol. Behav. 10, 443-449.

Schneider, G. E. (1970). Mechanisms of functional recovery following lesions of visual cortex or superior colliculus in neonate and adult hamsters. Brain Behav. Evol. 3, 295-323.

Stein, D. G., Rosen, J. J., Graziadei, J., Mishkin, D., and Brink, J. J. (1969). Central nervous system: Recovery of function. Science 166, 528-530.

Thompson, M. L., and Edwards, D. A. (1972). Olfactory bulb ablation and hormonally induced mating in spayed female mice. Physiol. Behav. 8, 1141-1146.

Vandenbergh, J. G. (1973). Effects of central and peripheral anosmia on reproduction of female mice. Physiol. Behav. 10, 257-261. 